\title{
Basic Life Support: Need of the Hour-A Study on the Knowledge of Basic Life Support among Young Doctors in India
}

\author{
Karapparambil Vineeth Chandran ${ }^{1}$, Siju V Abraham²
}

\begin{abstract}
Introduction: Knowledge about basic life support (BLS) is mandatory for healthcare professionals. This study aims to evaluate the knowledge among medical students who have completed their MBBS course and have enrolled for internship.

Materials and methods: A questionnaire pertaining to BLS before and after the BLS workshop (pretest and posttest 1) was distributed among the 50 participants consented for the study. The questionnaire was again given to the same participants at the end of their 1 year of internship (posttest 2) and were analyzed.

Results: Among the participants, $96 \%$ of students had attended nonstructured BLS classes in the past but the knowledge and skill in BLS and ability to recognize arrest were very low. Knowledge about the essential components of effective cardiopulmonary resuscitation (CPR) was poor among students, which improved to near $100 \%$ in posttest 1 . Awareness about cervical spine stabilization, log rolling, and management of choking was poor among the students, which improved after the class. Although attrition had occurred, the knowledge in posttest 2 was significantly better than pretest ( $p$ value $<0.05$ ).

Conclusion: The BLS knowledge among young doctors in India is very low as evidenced by poor performance in the pretest. Regular BLS courses are necessary to improve the knowledge among them and to prepare them to respond to a medical emergency.

Clinical significance: Knowledge and skills pertaining to BLS are not usually taught in medical schools in India. This study indicates the lack of knowledge among medical students about BLS and the need for improvement and regular update. This study can serve as a guide for policymakers to consider inculcating BLS knowledge into the medical school curriculum in the near future.

Keywords: Cardiopulmonary resuscitation, Chest compression, Knowledge, Medical education, Medical students, Training.

Indian Journal of Critical Care Medicine (2020): 10.5005/jp-journals-10071-23442
\end{abstract}

\section{INTRODUCTION}

Cardiac arrest is the sudden cessation of cardiac function resulting in respiratory and circulatory standstill. ${ }^{1}$ Most cardiac arrests in adults are sudden, resulting from a primary cardiac cause and hence, circulation produced by chest compressions is of paramount importance. ${ }^{1}$ The value of early cardiopulmonary resuscitation (CPR) is that it can buy time for the primary cardiac arrest patient by producing enough blood flow to the central nervous system and the myocardium to maintain temporary viability. To do so, however, CPR must be started early. ${ }^{2}$ Timely CPR improves the likelihood of survival by two to four times. ${ }^{2}$ Basic life support (BLS) comprises of the very basic tasks of resuscitation including CPR. The American Heart Association (AHA) guidelines for BLS include recognition and management of cardiac arrest, respiratory arrest, and choking in adults and the pediatric age group. ${ }^{3}$ Management of cardiac arrest includes a series of lifesaving interventions like immediate recognition of cardiac arrest and activation of the emergency response team, early CPR, rapid defibrillation, effective advanced life support, and integrated post cardiac arrest care. ${ }^{1}$ Further, BLS techniques have a global implication because of the fact that it requires nothing other than manpower as far as resources are concerned. Proper practice of the techniques and maneuvers enables a person to effectively resuscitate a victim. ${ }^{4}$

Awareness and understanding about the basic concepts of resuscitation is expected from every medical personnel involved in
${ }^{1}$ Department of Emergency Medicine, Aster MIMS, Calicut, Kerala, India

${ }^{2}$ Department of Emergency Medicine, Jubilee Mission Medical College and Research Institute, Thrissur, Kerala, India

Corresponding Author: Karapparambil Vineeth Chandran, Department of Emergency Medicine, Aster MIMS, Calicut, Kerala, India, Phone: +91 8281282351, e-mail: vineethchandran577@gmail.com

How to cite this article: Vineeth Chandran K, Abraham SV. Basic Life Support: Need of the Hour-A Study on the Knowledge of Basic Life Support among Young Doctors in India. Indian J Crit Care Med 2020;24(5):332-335.

Source of support: Nil

Conflict of interest: None

treating the patients. Researches have implicated poor awareness of BLS among doctors, interns, medical/dental students, and paramedics in the country. ${ }^{5-9}$ But unlike this study, only a few have followed up the BLS knowledge retention among the participants who have undergone the course.

\section{Objectives}

- Evaluate the knowledge and preparedness among interns in performing BLS and basic resuscitation skills.

() The Author(s). 2020 Open Access This article is distributed under the terms of the Creative Commons Attribution 4.0 International License (https://creativecommons. org/licenses/by-nc/4.0/), which permits unrestricted use, distribution, and non-commercial reproduction in any medium, provided you give appropriate credit to the original author(s) and the source, provide a link to the Creative Commons license, and indicate if changes were made. The Creative Commons Public Domain Dedication waiver (http://creativecommons.org/publicdomain/zero/1.0/) applies to the data made available in this article, unless otherwise stated. 
- Reassess knowledge retention after a period of 1 year among the same group of participants.

\section{Materials and Methods}

\section{Study Design}

This was a prospective cohort study conducted among young doctors who have completed their final year of medical education and have enrolled for their internship. A total of 100 candidates were approached, of which 50 consented to participate in the study.

\section{Variables Studied}

Participants were given a questionnaire pertaining to BLS (AHA guidelines 2015) and responses were collected. The questionnaire comprised of basic details like age, gender, and 15 questions regarding the knowledge in BLS. The questionnaire was filled in front of the course coordinator to prevent any malpractices. After the pretest, they were given a 5-hour class on BLS with skill training. The class was taken by BLS instructors approved by the AHA. The Class comprised of lectures and hands-on training on manikins. After the class, an immediate posttest (posttest 1) was performed with the same questionnaire and the responses were collected. After a period of 1 year, at the end of the internship, the questionnaire was again given to the group (posttest 2) and responses were collected. An analysis of the responses was done.

\section{Study Setting}

Jubilee Mission Medical College and Research Institute, Thrissur, Kerala, India.

\section{Study Population}

The MBBS students who have completed their course and have enrolled for internship.

\section{Sample Size}

Fifty

\section{Study Type}

Prospective cohort study.

\section{Statistical Analysis}

The collected data were coded and entered into Microsoft Excel to make a spreadsheet. Statistical analyzes were done using the Statistical Package Software for Social sciences (SPSS) version 20.0. The descriptive and frequency analysis has been done for counts, means, and percentages. A repeated measure of analysis of variance (ANOVA) with Bonferroni and McNemar tests was used to compare the measurements in the study. The $p$ value less than 0.05 was taken as the criteria for statistical significance.

\section{Results}

Of the total study population ( $n=50), 82 \%$ interns were females and $18 \%$ were males. About $96 \%$ of the group had attended some form of BLS classes in the past. In the pretest scenario, only $56 \%$ could recognize a cardiac arrest scenario and $28 \%$ were unsure of it. Immediately after the class, all the interns were confident in recognizing an arrest scenario. In the post 1-year test, still 86\% were sure of recognizing an arrest and $14 \%$ were unsure. In the pretest period, when the interns were just starting their internship, half of them (54\%) haven't seen a patient losing consciousness and only $8 \%$ had ever done a CPR. After 1 year of internship, 92\% had performed CPR and $88 \%$ had seen someone losing consciousness.

Interns were asked to scale their skills in various aspects related to BLS from a scale of 1 to 5 , with scale 1 being least and scale 5 being the highest. When interns were asked about their knowledge in BLS in the pretest session, the pretest score mean was $2.32 \pm 0.95$ and the immediate post-session score mean was $4.16 \pm 0.17$. Post 1 -year test, the mean value was $3.30 \pm 0.81$. A repeated measures of ANOVA determined that mean score of knowledge (1 to 5) differed statistically significant between different time intervals of the training period $(p=0.000)$. The post hoc test using Bonferroni revealed that there are statistically significant difference between pre-training and post-session ( $p=0.000)$; pre-training and post 1 year $(p=0.000)$; and post-session and post 1 year $(p=0.000)$ (Table 1).

The question regarding confidence in performing CPR also followed a similar trend. More than $90 \%$ of participants had rated their skill around scale 1 to 3 in the pretest period (mean value 2.30 \pm 0.83 ) whereas in posttest to a mean value of $4 \pm 0.75$, but post 1 year it was around scale 3 to $4(3.36 \pm 0.85)$. A repeated measure of ANOVA and the post hoc test using Bonferroni determined that mean score of performance (1 to 5 ) differed statistically significant between different time intervals of the training period $(p=0.000)$. With regards to skill in rescue breathing, in the pretest period, most of them were around scale 1 to 3 (mean $2.08 \pm 0.80$ ), immediate posttest to a mean of $4 \pm 0.88$, and post 1-year period, to around scale 2 to 4 (mean $3.08 \pm 0.92$ ). A repeated measures of ANOVA and the post hoc test using Bonferroni determined that mean score of rescue breathing ( 1 to 5 ) differed statistically significant between different time intervals of the training period $(p=0.000)$ (Table 1).

Questions were also asked regarding the theoretical knowledge about performing a CPR. Both in pretest and posttest periods, most of them were aware about the CPR ratio $30: 2$ (78\% in pretest vs $86 \%$ in post 1-year test) ( $p=0.049)$ (Table 2 ).

Only $22 \%$ had answered correctly regarding hand position in CPR in the pretest session, which improved to $76 \%$ posttest and $56 \%$ post 1-year test. The McNemar-Bowker test reveals that there is statistically significant difference in the knowledge of hand position in chest compression between pre-training and posttraining $(p=0.000)$; pre-training and post 1 year $(p=0.029)$; and post-training and post 1 year $(p=0.034)$ too.

Only $44 \%$ was aware about the actual required depth of compression in the pretest session as compared to $74 \%$ in post 1 -year session. Data reveal that all participants' answers regarding depth of chest compression in post-training session are $100 \%$ correct but after 1 year of training many answers were wrong. The McNemar test declared that there is statistically significant difference ( $p=0.028$ ) between pre and post 1-year session.

Among the group, $54 \%$ were aware about the $C A B$ sequence of resuscitation in the pretest whereas in post 1-year test, it increased only to $66 \%$ and around $26 \%$ opted for the $A B C$ sequence. The McNemar-Bowker test reveals that there is statistically significant difference in the knowledge of sequence of CPR between pretraining and post $(p=0.000)$; post-session and after 1 year of training $(p=0.001)$, but when the time goes, knowledge attrition occurs and shows no significant changes $(p=0.465)$ between pre and post 1 year of training.

In the pretest, only $56 \%$ were confident in helping a choking victim whereas in the post 1 -year test, it rose to $78 \%$. The McNemar 
Table 1: Level of confidence in performing basic resuscitation

\begin{tabular}{|c|c|c|c|c|c|c|c|c|c|c|c|c|c|c|c|}
\hline \multirow[b]{2}{*}{ Likert scale } & \multicolumn{5}{|c|}{ Pretest (\%) } & \multicolumn{5}{|c|}{ Posttest 1 (\%) } & \multicolumn{5}{|c|}{ Posttest 2 (\%) } \\
\hline & l & II & III & IV & V & I & II & III & IV & V & l & II & III & IV & V \\
\hline BLS knowledge & 22 & 34 & 36 & 6 & 2 & 0 & 0 & 18 & 48 & 34 & 2 & 8 & 56 & 26 & 8 \\
\hline CPR effectiveness & 18 & 40 & 36 & 6 & 0 & 0 & 0 & 28 & 44 & 28 & 0 & 14 & 46 & 30 & 10 \\
\hline Rescue breathing & 24 & 48 & 24 & 4 & 0 & 0 & 6 & 20 & 42 & 32 & 0 & 28 & 46 & 16 & 10 \\
\hline C spine stabilization & 64 & 24 & 6 & 2 & 4 & 2 & 12 & 28 & 42 & 16 & 8 & 24 & 30 & 26 & 12 \\
\hline Log roll & 74 & 14 & 6 & 2 & 4 & 2 & 12 & 30 & 32 & 24 & 6 & 8 & 36 & 28 & 22 \\
\hline
\end{tabular}

Table 2: Knowledge about CPR

\begin{tabular}{|c|c|c|c|c|c|c|c|c|c|}
\hline \multirow[b]{2}{*}{ Responses } & \multicolumn{3}{|c|}{ Pretest (\%) } & \multicolumn{3}{|c|}{ Posttest 1 (\%) } & \multicolumn{3}{|c|}{ Posttest 2 (\%) } \\
\hline & Correct & Wrong & Do not know & Correct & Wrong & Do not know & Correct & Wrong & Do not know \\
\hline Hand position & 22 & 44 & 34 & 76 & 22 & 2 & 56 & 32 & 12 \\
\hline CPR ratio & 78 & 6 & 16 & 100 & 0 & 0 & 86 & 6 & 8 \\
\hline Depth of chest compressions & 44 & 14 & 42 & 100 & 0 & 0 & 74 & 8 & 18 \\
\hline Frequency of chest compressions & 20 & 30 & 50 & 96 & 4 & 0 & 48 & 28 & 24 \\
\hline Resuscitation sequence & 54 & 30 & 16 & 96 & 2 & 2 & 66 & 28 & 6 \\
\hline
\end{tabular}

test reveals that there is statistically significant difference in the knowledge of helping chocking adult between pre-training and post $(p=0.000)$; pre-training and post 1 year $(p=0.015)$, but there is no significant change $(p=0.065)$ in their knowledge between post-session and after 1 year of training.

In the pretest period, most of the interns were unsure about the ways of stabilizing cervical spine and most of them were around scale 1 and 2 (mean $1.58 \pm 0.99$ ) in their knowledge on cervical spine stabilization. Post 1 year most of them were around scale 2 to scale 4 (mean $3.1 \pm 1.14$ ) A repeated measures of ANOVA determined that mean score of knowledge (1 to 5) in stabilizing a cervical spine of a trauma victim differed statistically significant between different time intervals of the training period $(p=0.000)$. The post hoc test using Bonferroni revealed that there are statistically significant difference between pre-training and post-session $(p=0.000)$; pretraining and post 1 year $(p=0.000)$; but no statistical significance between post-session and post 1 year $(p=0.048)$.

Knowledge about log roll was also poor among interns in the pretest period (mean $1.48 \pm 0.99$ ). About $74 \%$ were around scale 1 in the pretest period whereas in post 1-year test, most of them were around scale 3 to 5 (mean $3.52 \pm 0.11$ ) (Table 1). Finally, all students were willing to attend courses to improve their basic resuscitation skills.

\section{Discussion}

This study was intended to assess the knowledge of interns about BLS and to reassess them after giving training. As the study shows, though most of them had attended some BLS training previously, the knowledge about BLS was poor. According to previous studies, the knowledge about BLS among medical students across the subcontinent was found to be low. ${ }^{4-6}$ The results of this study also showed a similar trend. But the knowledge and confidence in performing BLS among the interns in this study improved steeply after attending a structured course on BLS. The students were confident enough to recognize a cardiac arrest and to perform necessary lifesaving procedures and to help a choking victim. They also gained confidence in basic trauma assessment and management procedures like cervical spine stabilization and log rolling. The study also points toward the necessity of reinforcing the knowledge from time to time to prevent skill and knowledge attrition. Studies have also stressed the importance of reinforcement to attain adequate CPR skills and maintain continued competency in the technique. ${ }^{7,8}$ Also, it is necessary to update the knowledge regularly. In our study, posttest 2 showed that students were better equipped with managing a cardiac arrest scenario but had attrition in knowledge and skills over the course of 1 year. Therefore, this calls up for a recurrent revision and retraining in the skills.

According to Pande et al., the proper time seems to be the incorporation of BLS training in the medical curriculum in the first year itself and the reinforcement of the skills every year. ${ }^{9}$ After graduation, there is hardly any time for students to practice the skill. The right time to foster any skill is during graduation. Zaheer et al. also concluded that the inclusion of a BLS course will increase awareness and application of this valuable lifesaving maneuver. ${ }^{4}$ Therefore, BLS and advanced cardiovascular life support training programs should be mandatory for all medical and paramedical staff. Educating medical undergraduates about BLS may turn out to be an excellent strategy to reach the community at large if students share the acquired knowledge with their families and friends.

\section{Limitations of the Study}

The study population is very small and is a single-center study. The study also only includes fresh MBBS graduates. A larger group of population including nursing and paramedic staff and practising doctors will help find out the actual state of BLS knowledge among medical community. Also, this study only assesses knowledge and not BLS performance skills, which is a major limiting factor.

\section{Conclusion}

The BLS knowledge among young doctors in India is very low as evidenced by poor performance in the pretest.

Regular BLS courses with frequent follow-up classes are necessary to improve the knowledge, to prepare them to respond to a medical emergency, and also to prevent knowledge attrition among them. 


\section{Clinical Significance}

Knowledge about BLS is mandatory for health professionals. This study shows that knowledge about BLS and basic resuscitation skills are poor among young doctors in a tertiary care center in southern part of India. A suggestion would be to include these skills in undergraduate medical curriculum. Also, this study signifies the need for regular update classes and mock drills to help students retain their knowledge and skill sets.

\section{REFERENCES}

1. Travers AH, Rea TD, Bobrow BJ, Edelson DP, Berg RA, Sayre MR, et al. Part 4: CPR Overview: 2010 American Heart Association Guidelines for Cardiopulmonary Resuscitation and Emergency Cardiovascular Care. Circulation 2010;122(18_suppl_3):S676-S684. DOI: 10.1161/ CIRCULATIONAHA.110.970913.

2. Cummins R, Ornato J, Thies W, Pepe P. Improving survival from sudden cardiac arrest: the 'chain of survival' concept. A statement for health professionals from the Advanced Cardiac Life Support Subcommittee and the Emergency Cardiac Care Committee, American Heart Association. Circulation. 1991;83(5):1832-1847.
3. Hazinki M. BLS for healthcare providers. Dallas, Tex.: American Heart Association; 2011.

4. Zaheer $\mathrm{H}$, Haque Z. Awareness about BLS (CPR) among medical students: status and requirements. J Pak Med Assoc 2009;5(1):57-59.

5. Chandrasekaran S, Kumar S, Bhat SA, Saravanakumar, Shabbir PM, Chandrasekaran V. Awareness of basic life support among medical, dental, nursing students and doctors. Indian J Anaesth 2010;54(2):121-126. DOI: 10.4103/0019-5049.63650.

6. Chaudhary A, Parikh H, Dave V. Current scenario: knowledge of basic life support in medical college. Natl J Med Res 2011;1: 80-82.

7. Cooper S, Johnston E, Priscott D. Immediate life support (ILS) training. Impact in a primary care setting? Resuscitation. 2007;72(1):92-99. DOI: 10.1016/j.resuscitation.2006.06.004.

8. Roshana S, Batajoo KH, Piryani RM, Sharma MW. Basic life support: knowledge and attitude of medical/paramedical professionals. World J Emerg Med 2012;3(2):141-144. DOI: 10.5847/wjem.j.i ssn.1920-8642.2012.02.011.

9. Pande S, Pande S, Parate V, Pande S, Sukhsohale N. Evaluation of retention of knowledge and skills imparted to first-year medical students through basic life support training. Adv Physiol Educ 2014;38(1):42-45. DOI: 10.1152/advan.00102.2013. 